\title{
Localização coerente passiva de um alvo usando redes neurais feedforward
}

\author{
Daniel P. Nicolalde-Rodríguez, Wallace A. Martins, José A. Apolinário Jr. e Luiz P. Calôba
}

Resumo-Este trabalho aborda a localização coerente passiva de um alvo num cenário com um transmissor e vários receptores. Num primeiro caso, uma rede neural feedforward estima a localização de um alvo com base nas diferenças de tempo de chegada entre os sinais captados pelos receptores, cujas posições são fixas e conhecidas, após serem refletidos pelo alvo. Num segundo caso, a rede neural considera também as posições dos receptores como variáveis de entrada para computar a posição do alvo. Em ambos os casos, a localização do transmissor é fixa. São apresentados a formulação do problema, os métodos usados e os resultados obtidos. Simulações em cenários com diferentes quantidades de receptores e diferentes graus de erro foram realizadas para assim determinar os parâmetros das redes.

Palavras-Chave-Localização coerente passiva (PCL), rede neural feedforward, radar passivo, localização do alvo, diferença de tempo de chegada (TDOA).

Abstract-This work addresses the passive coherent location of a target in a scenario with a single transmitter and several receivers. Firstly, a feedforward neural network estimates the target location based on the time differences of arrival among the target-reflected signals acquired by the receivers, whose positions are fixed and known. In a second case, the neural network also considers the receivers' positions as inputs to compute the target position. In both cases, the transmitter has a fixed location. Problem formulation, methods employed, as well as corresponding simulation results are presented. The simulations consider scenarios with different numbers of receivers and different degrees of errors to determine the network parameters.

Keywords-Passive coherence location (PCL), feedforward neural network, passive radar, target positioning, time difference of arrival (TDOA).

\section{INTRODUÇÃO}

Em um sistema com vários transmissores e receptores, no contexto de localização coerente passiva (PCL, do inglês passive coherent location), também conhecido como radar passivo, é possível estimar a localização de um alvo a partir dos sinais refletidos no alvo e dos sinais em visada direta entre cada par transmissor (TX) e receptor (RX) [1]. Várias pesquisas têm focado no parâmetro da diferença de tempo de chegada (TDOA, do inglês time difference of arrival) entre o sinal de cada par TX-RX e o refletido no alvo [2]-[7].

Malanowski em [4] propõe dois métodos que, baseados nas TDOAs de pares TX-RX, computam a localização de um alvo usando fórmulas fechadas: interpolação esférica (SI, do inglês spherical interpolation) e intersecção esférica (SX, do inglês

D.P. Nicolalde-Rodríguez, W.A. Martins e L.P. Calôba, PEE/COPPE, Universidade Federal de Rio de Janeiro, Rio de Janeiro, RJ, Brasil, E-mails: danielnicolalde@gmail.com,wallace.martins@smt.ufrj.br e lcaloba@gmail.com. J.A. Apolinário Jr., Programa de Pósgraduação em Engenharia Elétrica, Instituto Militar de Engenharia, Rio de Janeiro, RJ, Brasil, E-mail: apolin@ime.eb.br. Este trabalho foi financiado por Capes e Faperj. spherical intersection). Tais métodos não consideram possíveis erros de estimação dos TDOAs, nem erros nas posições dos RXs e TXs. Na prática, estes erros podem ser críticos para a localização final. Em [7], uma modificação do algoritmo SI melhora a acurácia e a precisão na estimativa da posição adicionando uma restrição não linear que leva em conta os erros das medidas de TDOA. O método é denominado NLCLS (do inglês nonlinearly constrained least squares).

Redes neurais (RNs) também têm sido utilizadas em sistemas de localização, uma vez que possuem alta capacidade de aproximar funções não lineares, característica útil para a aplicação em questão. Por exemplo, os autores em [8] usam RNs para estimar a localização de um terminal móvel usando as TDOAs entre o terminal e as estações-base circundantes. Por outro lado, as RNs, quando treinadas em ambientes ruídosos, podem melhorar a acurácia e a precisão na estimativa final em tais ambientes. No contexto de PCL, uma RN é proposta em [9] para computar a localização de um alvo num ambiente com um RX e múltiplos TXs, levando em conta os TDOAs dos pares TX-RX em diferentes condições de ruído.

Neste trabalho, consideramos RNs para estimar a localização de um alvo, em duas dimensões (2-D), levando em conta diferentes graus de erro nas estimativas de TDOAs. A localização é feita para o caso de 1 TX e vários RXs, com posições fixas e conhecidas. Além desse cenário, estendemos a abordagem para um segundo caso onde as posições dos RXs são variáveis de entrada da rede, aumentando assim sua complexidade. Experimentos exaustivos são realizados em diferentes cenários com vários RXs em diferentes localizações e diferentes posições de alvo. Também, são feitas comparações com os métodos SI, SX [4] e NLCLS [7].

$\mathrm{O}$ presente trabalho foi organizado da seguinte maneira. $\mathrm{Na}$ Seção II são apresentados a definição do problema e o método proposto. Os resultados experimentais são mostrados na Seção III e as conclusões são discutidas na Seção IV.

\section{O Problema e o Método Proposto}

\section{A. Definição do problema}

Consideramos aqui um sistema PCL com 1 TX e $M$ RXs, cuja geometria é apresentada na Fig. 1. Temos $M$ TDOAs para uma dada posição do alvo. O TDOA associado ao $m$-ésimo par TX-RX é denotado por $\tau_{m}, \operatorname{com} m \in \mathfrak{M} \triangleq\{1,2, \ldots, M\}$.

No sistema de localização consideramos $P_{\mathrm{r}}$ posições teóricas aleatórias uniformes de um alvo que pode se deslocar livremente num quadrado de $(20 \times 20) \mathrm{km}^{2}$. Neste quadrado, temos um TX fixo localizado em $(7 \mathrm{~km}, 0)$, e $M$ RXs posicionados em regiões aleatórias (uniformes) que fazem parte de 


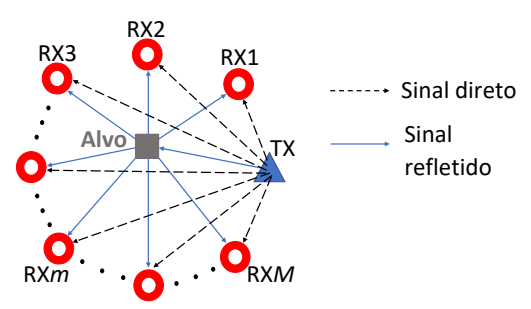

Fig. 1: Geometria de um radar passivo com $1 \mathrm{TX}$ e $M$ RXs.

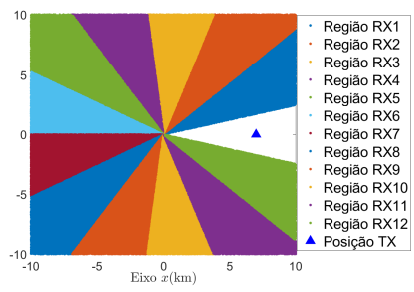

(a)

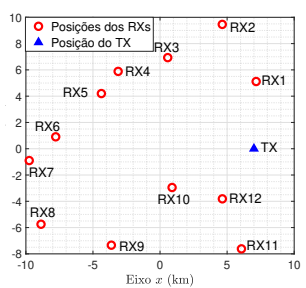

(b)
Fig. 2: Sistema com 12 RXs e 1 TX. (a) Regiões de localização dos RXs e localização fixa do TX. (b) Exemplo com 12 RXs fixos.

uma grade angular e radial como a ilustrada na Fig. 2a para o caso de $M=12 \mathrm{RXs}$. Cada região angular começa no ponto $(0,0)$ com um ângulo de $\frac{2 \pi}{M+1}$ rad. A distância mínima entre os RXs é $\frac{10}{M} \mathrm{~km}$. A ideia principal é espalhar os RXs e evitar que fiquem muito próximos.

Nos nossos experimentos, consideramos dois cenários para a RN que estima a localização do alvo:

1) Posições dos RXs fixas: As entradas da rede são as TDOAs. A saída da rede é a posição do alvo.

2) Posições dos RXs variáveis: As entradas são as TDOAs e as posições dos RXs. A saída é a posição do alvo.

Neste trabalho, a posição fixa do TX foi mantida fora da entrada da rede visando uma menor complexidade computacional. Para os dois cenários, começamos abordando o préprocessamento assim como o escalamento apropriado das variáveis. Posteriormente, explicamos os diferentes blocos do sistema para finalmente expor o modelo de RN.

\section{B. Pré-processamento}

Para o sistema com RXs fixos, o vetor de entrada é $\boldsymbol{v}_{\text {in }}=$ $\left[\tau_{1} \ldots \tau_{M}\right]^{\top}$, contendo apenas TDOAs, enquanto que o vetor de saída, a localização estimada do alvo, é $\boldsymbol{v}_{\text {out }}=\left[\begin{array}{ll}x_{\mathrm{T}} & y_{\mathrm{T}}\end{array}\right]^{\top}$.

Avaliamos o coeficiente de correlação de Pearson ${ }^{1}$ entre entradas $r_{\tau_{m} \tau_{n}}$, e entradas e saídas $r_{\tau_{m} s}$, com $s \in \mathcal{S} \triangleq$ $\left\{x_{\mathrm{T}}, y_{\mathrm{T}}\right\}$, para verificar as entradas (RXs) significativas no sistema. A TABELA I apresenta os coeficientes para o sistema de 12 RXs mostrado na Fig. 2b (Análises similares podem ser feitas para outras localizações de RXs). Existe informação redundante proporcionada pelos RXs mais próximos entre si (Na TABELA I destacamos em negrito $\left|r_{\tau_{i} \tau_{j}}\right| \geq 0,88$ ). Quanto às saídas, quase todas as TDOAs proveem informação relevante para $x_{\mathrm{T}}$ ou $y_{\mathrm{T}}\left(\right.$ e.g. $\left.\left|r_{\tau_{m} s}\right| \geq 0,1\right)$.

${ }^{1}$ É usado para medir a correlação linear entre duas variáveis. É definido por $r_{x y}=\frac{C_{x y}}{\sigma_{x} \sigma_{y}}$, em que $C_{x y}$ é a covariância das variáveis $x$ e $y$, ao passo que $\sigma_{x}$ e $\sigma_{y}$ são os desvios padrões de $x$ e $y$, respectivamente.
TABELA I: Matriz de coeficientes de correlação de Pearson do sistema da Fig. $2 \mathrm{~b}$ para diferentes posições $\left(x_{\mathrm{T}}, y_{\mathrm{T}}\right)$ do alvo

\begin{tabular}{c|c|c|c|c|c|c|c|c|c|c|c|c|c|c} 
& $\tau_{1}$ & $\tau_{2}$ & $\tau_{3}$ & $\tau_{4}$ & $\tau_{5}$ & $\tau_{6}$ & $\tau_{7}$ & $\tau_{8}$ & $\tau_{9}$ & $\tau_{10}$ & $\tau_{11}$ & $\tau_{12}$ & $x_{\mathrm{T}}$ & $y_{\mathrm{T}}$ \\
\hline$\tau_{1}$ & $\mathbf{1 , 0 0}$ & $\mathbf{0 , 9 6}$ & $\mathbf{0 , 9 1}$ & 0,77 & 0,69 & 0,36 & 0,14 & 0,00 & 0,29 & 0,75 & 0,69 & 0,80 & $-0,86$ & $-0,35$ \\
\hline$\tau_{2}$ & $\mathbf{0 , 9 6}$ & $\mathbf{1 , 0 0}$ & $\mathbf{0 , 9 7}$ & 0,86 & 0,76 & 0,37 & 0,11 & $-0,16$ & 0,08 & 0,60 & 0,50 & 0,64 & $-0,76$ & $-0,57$ \\
\hline$\tau_{3}$ & $\mathbf{0 , 9 1}$ & $\mathbf{0 , 9 7}$ & $\mathbf{1 , 0 0}$ & $\mathbf{0 , 9 5}$ & $\mathbf{0 , 8 9}$ & 0,54 & 0,28 & $-0,06$ & 0,12 & 0,62 & 0,45 & 0,61 & $-0,62$ & $-0,59$ \\
\hline$\tau_{4}$ & 0,77 & 0,86 & $\mathbf{0 , 9 5}$ & $\mathbf{1 , 0 0}$ & $\mathbf{0 , 9 8}$ & 0,71 & 0,46 & 0,01 & 0,09 & 0,55 & 0,30 & 0,49 & $-0,40$ & $-0,63$ \\
\hline$\tau_{5}$ & 0,69 & 0,76 & $\mathbf{0 , 8 9}$ & $\mathbf{0 , 9 8}$ & $\mathbf{1 , 0 0}$ & 0,83 & 0,61 & 0,17 & 0,19 & 0,58 & 0,31 & 0,49 & $-0,29$ & $-0,53$ \\
\hline$\tau_{6}$ & 0,36 & 0,37 & 0,54 & 0,71 & 0,83 & $\mathbf{1 , 0 0}$ & $\mathbf{0 , 9 4}$ & 0,59 & 0,44 & 0,56 & 0,25 & 0,39 & 0,05 & $-0,15$ \\
\hline$\tau_{7}$ & 0,14 & 0,11 & 0,28 & 0,46 & 0,61 & $\mathbf{0 , 9 4}$ & $\mathbf{1 , 0 0}$ & 0,79 & 0,57 & 0,51 & 0,22 & 0,32 & 0,21 & 0,12 \\
\hline$\tau_{8}$ & 0,00 & $-0,16$ & $-0,06$ & 0,01 & 0,17 & 0,59 & 0,79 & $\mathbf{1 , 0 0}$ & $\mathbf{0 , 8 8}$ & 0,58 & 0,44 & 0,43 & 0,11 & 0,65 \\
\hline$\tau_{9}$ & 0,29 & 0,08 & 0,12 & 0,09 & 0,19 & 0,44 & 0,57 & $\mathbf{0 , 8 8}$ & $\mathbf{1 , 0 0}$ & 0,82 & 0,78 & 0,74 & $-0,31$ & 0,69 \\
\hline$\tau_{10}$ & 0,75 & 0,60 & 0,62 & 0,55 & 0,58 & 0,56 & 0,51 & 0,58 & 0,82 & $\mathbf{1 , 0 0}$ & $\mathbf{0 , 9 2}$ & $\mathbf{0 , 9 7}$ & $-0,67$ & 0,24 \\
\hline$\tau_{11}$ & 0,69 & 0,50 & 0,45 & 0,30 & 0,31 & 0,25 & 0,22 & 0,44 & 0,78 & $\mathbf{0 , 9 2}$ & $\mathbf{1 , 0 0}$ & $\mathbf{0 , 9 7}$ & $-0,80$ & 0,40 \\
\hline$\tau_{12}$ & 0,80 & 0,64 & 0,61 & 0,49 & 0,49 & 0,39 & 0,32 & 0,43 & 0,74 & $\mathbf{0 , 9 7}$ & $\mathbf{0 , 9 7}$ & $\mathbf{1 , 0 0}$ & $-0,81$ & 0,23
\end{tabular}

TABELA II: Matriz de coeficientes de correlação com variáveis de entrada descorrelaciondas para o sistema da Fig. 2b

\begin{tabular}{c|c|c|c|c|c|c|c|c|c|c|c|c|c|c} 
& $\tau_{1}$ & $\tau_{2}^{*}$ & $\tau_{3}$ & $\tau_{4}$ & $\tau_{5}^{*}$ & $\tau_{6}$ & $\tau_{7}$ & $\tau_{8}^{*}$ & $\tau_{9}$ & $\tau_{10}$ & $\tau_{11}$ & $\tau_{12}^{*}$ & $x_{\mathrm{T}}$ & $y_{\mathrm{T}}$ \\
\hline$\tau_{1}$ & $\mathbf{1 , 0 0}$ & $\mathbf{0 , 0 0}$ & $\mathbf{0 , 9 1}$ & 0,77 & - & 0,36 & 0,14 & - & 0,29 & 0,75 & 0,69 & - & $-0,86$ & $-0,35$ \\
\hline$\tau_{2}^{*}$ & $\mathbf{0 , 0 0}$ & $\mathbf{1 , 0 0}$ & - & - & - & - & - & - & - & - & - & - & $\mathbf{0 , 2 7}$ & $\mathbf{- 0 , 8 6}$ \\
\hline$\tau_{3}$ & $\mathbf{0 , 9 1}$ & - & $\mathbf{1 , 0 0}$ & $\mathbf{0 , 9 5}$ & - & 0,54 & 0,28 & - & 0,12 & 0,62 & 0,45 & - & $-0,62$ & $-0,59$ \\
\hline$\tau_{4}$ & 0,77 & - & $\mathbf{0 , 9 5}$ & $\mathbf{1 , 0 0}$ & $\mathbf{0 , 0 0}$ & 0,71 & 0,46 & - & 0,09 & 0,55 & 0,30 & - & $-0,40$ & $-0,63$ \\
\hline$\tau_{5}^{*}$ & - & - & - & $\mathbf{0 , 0 0}$ & $\mathbf{1 , 0 0}$ & - & - & - & - & - & - & - & $\mathbf{0 , 4 6}$ & $\mathbf{0 , 4 1}$ \\
\hline$\tau_{6}$ & 0,36 & - & 0,54 & 0,71 & - & $\mathbf{1 , 0 0}$ & $\mathbf{0 , 9 4}$ & - & 0,44 & 0,56 & 0,25 & - & 0,05 & $-0,15$ \\
\hline$\tau_{7}$ & 0,14 & - & 0,28 & 0,46 & - & $\mathbf{0 , 9 4}$ & $\mathbf{1 , 0 0}$ & $\mathbf{0 , 0 0}$ & 0,57 & 0,51 & 0,22 & - & 0,21 & 0,12 \\
\hline$\tau_{8}^{*}$ & - & - & - & - & - & - & $\mathbf{0 , 0 0}$ & $\mathbf{1 , 0 0}$ & - & - & - & - & $-\mathbf{0 , 1 0}$ & $\mathbf{0 , 9 0}$ \\
\hline$\tau_{9}$ & 0,29 & - & 0,12 & 0,09 & - & 0,44 & 0,57 & - & $\mathbf{1 , 0 0}$ & 0,82 & 0,78 & - & $-0,31$ & 0,69 \\
\hline$\tau_{10}$ & 0,75 & - & 0,62 & 0,55 & - & 0,56 & 0,51 & - & 0,82 & $\mathbf{1 , 0 0}$ & $\mathbf{0 , 9 2}$ & - & $-0,67$ & 0,24 \\
\hline$\tau_{11}$ & 0,69 & - & 0,45 & 0,30 & - & 0,25 & 0,22 & - & 0,78 & $\mathbf{0 , 9 2}$ & $\mathbf{1 , 0 0}$ & $\mathbf{0 , 0 0}$ & $-0,80$ & 0,40 \\
\hline$\tau_{12}^{*}$ & - & - & - & - & - & - & - & - & - & - & $\mathbf{0 , 0 0}$ & $\mathbf{1 , 0 0}$ & $-\mathbf{0 , 1 3}$ & $-\mathbf{0 , 7 1}$
\end{tabular}

Escolhemos pares de entradas (4 pares de TDOA neste caso), associados a pares de RXs bem próximos e com TDOAs com alta correlação entre si. Para cada par, descorrelacionamos uma variável (TDOA de um RX) com respeito à outra. Caso a nova variável descorrelacionada apresente valores baixos de correlação com respeito às saídas $x_{\mathrm{T}}$ e $y_{\mathrm{T}}$, podemos descartar o TDOA original. Desta maneira, definimos novas variáveis descorrelacionadas: $\tau_{2}^{*}$ equivalente a $\tau_{2}$ descorrelacionado de $\tau_{1}, \tau_{5}^{*}$ equivalente a $\tau_{5}$ descorrelacionado de $\tau_{4}, \tau_{8}^{*}$ equivalente a $\tau_{8}$ descorrelacionado de $\tau_{7}$ e $\tau_{12}^{*}$ equivalente a $\tau_{12}$ descorrelacionado de $\tau_{11}$. Na TABELA II apresentamos a matriz de correlação com estas novas variáveis descorrelacionadas ${ }^{2}$.

Verificamos que as novas variáveis descorrelacionadas ainda proporcionam informação importante para, ao menos, uma das saídas. Assim, a escolha do número de TDOAs de entrada (equivalente ao número de RXs) será feita por via de experimentos exaustivos como explicado na Seção III-A.

Para o sistema com posições dos RXs variáveis, o vetor

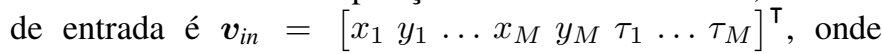
$x_{m}$ e $y_{m}$ representam a localização no eixos $x$ e $y$ de $\mathrm{RX} m$. O vetor de saída continua $\boldsymbol{v}_{\text {out }}=\left[\begin{array}{ll}x_{\mathrm{T}} & y_{\mathrm{T}}\end{array}\right]^{\top}$. Dado o número de variáveis e os diferentes cenários, o sistema é mais complexo. Na matriz de coeficientes de correlação de Pearson (não incluída neste artigo considerando o tamanho da matriz), observamos informação redundante entre TDOAs pertencentes principalmente a RXs vizinhos e pouca informação redundante entre as localizações dos RXs. Quanto às saídas, verificamos

\footnotetext{
${ }^{2}$ Levando em conta que $\tau_{i}^{*}$ representa $\tau_{i}$ descorrelacionado de $\tau_{j}$, computamos o coeficiente de correlação de $\tau_{i}^{*}$ com respeito à saída $x$ como: $r_{i * x}=\frac{r_{i x}-r_{j x} r_{i j}}{\sqrt{1-r_{i j}^{2}}}$, em que $r_{i j}$ é o coeficiente entre $\tau_{i}$ e $\tau_{j}$ e $r_{i x}$ é o coeficiente entre $\tau_{i}$ e $x$.
} 


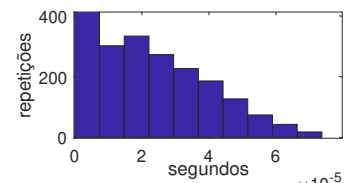

(a)

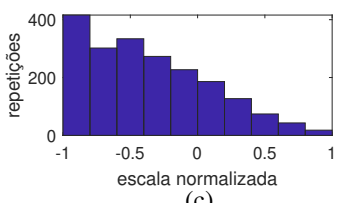

(c)

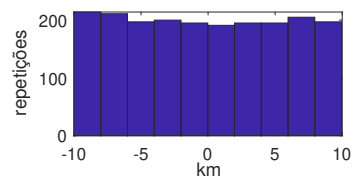

(b)

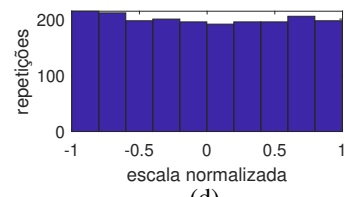

(d)
Fig. 3: Histograma de (a) $\tau_{5}$, (b) $y_{\mathrm{T}}$, (c) $\tau_{5}^{\prime}$ e (d) $y_{\mathrm{T}}^{\prime}$ para a Fig $2 \mathrm{~b}$

uma correlação forte com respeito às TDOAs e uma baixa correlação em relação às localizações dos RXs. Mesmo assim, as localizações dos RXs são importantes para outorgar a diversidade da RN. Tal como no primeiro caso, o número ótimo de variáveis de entrada será avaliado na Seção III-A.

Para garantir um processamento numérico correto da rede, escalamos todas as variáveis para o intervalo $[-1,1]$. As variáveis escaladas são: $\tau_{m}^{\prime}\left(\tau_{m}\right.$ escalada $),\left(x_{m}^{\prime}, y_{m}^{\prime}\right)\left(\left(x_{m}, y_{m}\right)\right.$ escaladas $)$, e $\left(x_{\mathrm{T}}^{\prime}, y_{\mathrm{T}}^{\prime}\right)\left(\left(x_{\mathrm{T}}, y_{\mathrm{T}}\right)\right.$ escaladas $)$. A título de exemplo, a Fig. 3 apresenta histogramas das variáveis originais do sistema da Fig. 2 b, $\left(\tau_{5}, y_{\mathrm{T}}\right)$, e suas versões escaladas, $\left(\tau_{5}^{\prime}, y_{\mathrm{T}}^{\prime}\right)$.

\section{A localização do alvo}

$\stackrel{\boldsymbol{v}_{\text {in }}}{\longrightarrow}$ Escalador $\stackrel{\boldsymbol{v}_{\text {in }}^{\prime}}{\longrightarrow}$ REDE NEURAL $\stackrel{\widehat{\boldsymbol{v}}_{\text {out }}^{\prime}}{\longrightarrow}$ Desescalador $\stackrel{\widehat{\boldsymbol{v}}_{\text {out }}}{\longrightarrow}$

Fig. 4: Diagrama de blocos do sistema de localização do alvo.

O sistema de localização para os dois casos, RXs com localizações fixas e RXs com localizações variáveis, tem três blocos, tal como apresentado na Fig. 4. O primeiro bloco é o escalador que normaliza as variáveis do $\boldsymbol{v}_{\text {in }}$ na escala de -1 a 1. Este bloco cria o vetor de entrada escalado $\boldsymbol{v}_{i n}^{\prime}$. O segundo bloco é a RN feedforward que obtém na saída o vetor da estimativa da localização escalada do alvo $\hat{\boldsymbol{v}}_{\text {out }}^{\prime}=\left[\hat{x}_{\mathrm{T}}^{\prime} \hat{y}_{\mathrm{T}}^{\prime}\right]^{\top}$. Finalmente, o desescalador retornará $\hat{\boldsymbol{v}}_{\text {out }}^{\prime}$ à escala original, obtendo-se assim a localização final do alvo $\hat{\boldsymbol{v}}_{\text {out }}=\left[\begin{array}{ll}\hat{x}_{\mathrm{T}} & \hat{y}_{\mathrm{T}}\end{array}\right]^{\top}$.

\section{Modelo de rede para estimar a localização do alvo}

Usamos a RN feedforward, como aproximador universal, com modelo para mapeamento não-linear com duas camadas. Uma camada oculta, com $L$ neurônios, que usa como função de ativação da saída de cada neurônio a função tangente hiperbólica, e uma camada de saída, com 2 neurônios, com função de ativação linear. A Fig. 5 apresenta o modelo usado.

$\mathrm{O}$ vetor de entrada da rede para o caso do sistema com RXs fixos é $\hat{\boldsymbol{v}}_{i n}^{\prime}=\left[\tau_{1}^{\prime} \ldots \tau_{M}^{\prime}\right]^{\top}$ e para o caso do sistema com RXs

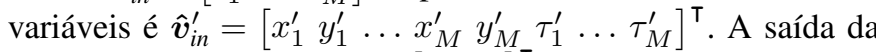
rede computa o vetor $\hat{\boldsymbol{v}}_{\text {out }}^{\prime}=\left[\begin{array}{ll}\hat{x}_{\mathrm{T}}^{\prime} & \hat{y}_{\mathrm{T}}^{\prime}\end{array}\right]^{\top}$, e $w_{l k}$ e $t_{n l}$ representam as sinapses das camadas oculta e de saída, respectivamente.

No treinamento da RN, minimizamos o erro quadrático médio da saída estimada da rede, tendo como referência a posição teórica do alvo: $F_{0}=\frac{1}{P} \sum_{k=1}^{P} \epsilon_{k}^{2}$, onde $\epsilon_{k}^{2}=\left\|\boldsymbol{v}_{\text {out }}^{\prime{ }^{k}}-\hat{\boldsymbol{v}}_{\text {out }}^{k^{\prime}}\right\|^{2}$

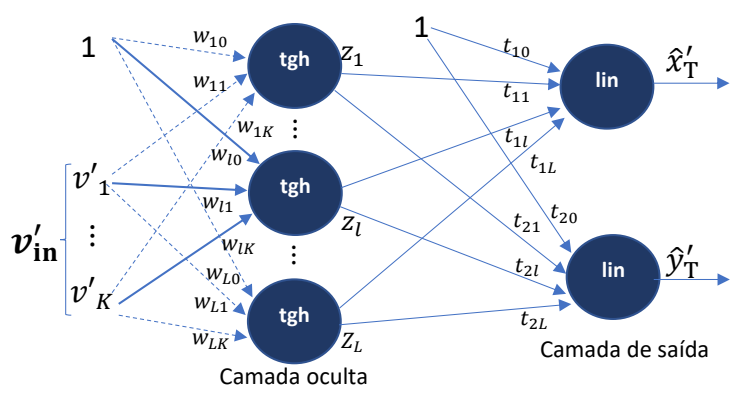

Fig. 5: Modelo da RN para estimar a localização de um alvo.

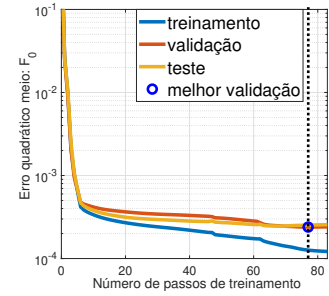

(a)

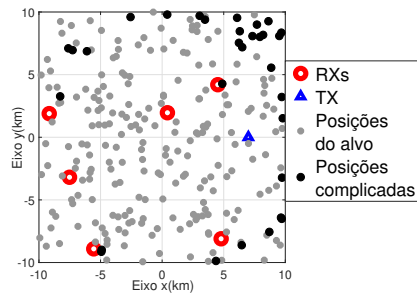

(b)
Fig. 6: Sistema com 12 RXs e 1 TX. (a) Regiões de localização dos RXs e localização fixa do TX. (b) Exemplo com 12 RXs fixos.

representa a norma quadrática da diferença entre $\boldsymbol{v}_{\text {out }}^{\prime k}$ (teórico) e $\hat{\boldsymbol{v}}_{\text {out }}^{\prime^{\prime}}$ (estimado) para o $k$-ésimo par de vetores (entradasaída). $P$ representa o número total de pares. Consideramos o algoritmo Levenberg-Marquadt [10] para treinar a rede, levando em conta um grupo dos pares de vetores (entradasaída) para treinamento, um outro para teste e um outro para validação.

\section{RESUltAdos EXPERIMENTAIS}

Todos os experimentos são realizados com base em $P_{\mathrm{r}}$ posições aleatórias do alvo, uniformemente distribuídas, num quadrado de $(20 \times 20) \mathrm{km}^{2}$, onde temos um TX com posição fixa $(7 \mathrm{~km}, 0)$ e $M$ RXs localizados em regiões separadas, como explicado na Seção II-A. Para cada posição do alvo, somamos a cada $\tau_{m}$ teórico ruído branco gaussiano com média zero e desvio-padrão $\sigma_{E} \in \mathcal{E} \triangleq\{0,1,2,3,4\} \%$ do próprio valor de $\tau_{m}$. Obtemos ao total $P_{\mathrm{r}}$ pares de vetores (entradasaída) para criar a rede. Estes pares foram distribuídos com $P_{\text {trein }}=P_{\mathrm{r}} / 2$ para treinamento, $P_{\text {teste }}=P_{\mathrm{r}} / 4$ para teste e $P_{\text {val }}=P_{\mathrm{r}} / 4$ para validação.

Um caso do procedimento de treinamento de uma $\mathrm{RN}$ com 6 RXs fixos, projetada com $L=15$ neurônios e $P_{\mathrm{r}}=1000$ pares $\left(P_{\text {trein }}=500\right.$ e $\left.P_{\text {teste }}=P_{\text {val }}=250\right)$, é apresentado na Fig. 6a. Na evolução do treinamento, onde progressivamente se diminui $F_{0}$, a rede final corresponde ao passo 77 , correspondente ao melhor valor de $F_{0}$ para o conjunto de validação: $F_{0}=2.3937 \times 10^{-4}$ (evitando o overtraining da rede).

Para um correto funcionamente da rede é necessário verificar a quantidade ótima de: neurônios $L, M$-RXs (TDOAs), e os pares $P_{\mathrm{r}}$. Desta forma, devemos avaliar o desempenho do sistema quando adicionado ruído branco. 


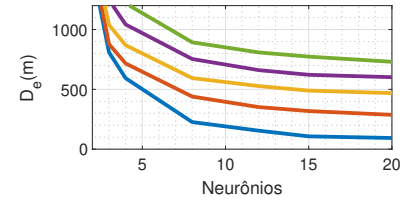

(a)

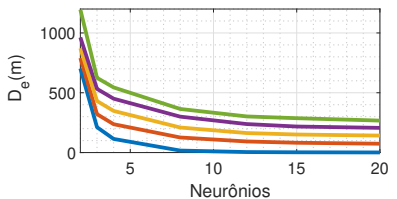

(c)

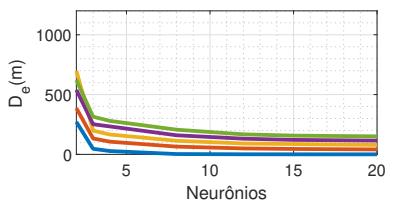

(e)

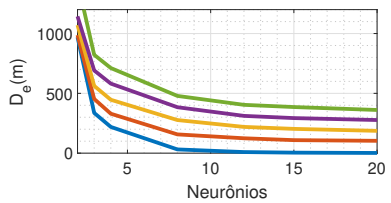

(b)

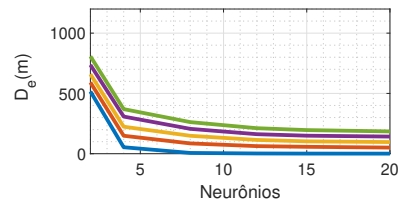

(d)

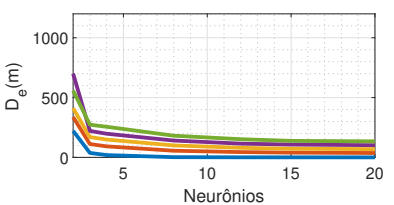

(f)

$$
-0 \%-1 \%-2 \%-3 \%-4 \%
$$

Fig. 7: Distância de erro médio total, $D_{\mathrm{e}}$ vs neurônios, $L$, para rede com $M$ RXs fixos: $P_{\mathrm{r}}=4000: P_{\text {trein }}=2000, P_{\text {teste }}=1000$, $P_{\text {val }}=1000$ e $\sigma_{E}$ variando de 0 a $4 \%$ de $\tau_{m}$. (a) $M=3$, (b) $M=$ 4, (c) $M=6$, (d) $M=10$, (e) $M=15$, (f) $M=20$.

\section{A. Dimensionamento da rede neural}

Para a RN com RXs fixos, fizemos experimentos exaustivos para: $P_{\mathrm{r}} \in \mathcal{P} \triangleq\{400,1000,2000,4000\}$ pares, $M \in \mathcal{M} \triangleq$ $\{3,4,6,10,15,20\}$ RXs, $L \in \mathcal{L} \triangleq\{2,3, \ldots, 20\}$ e $\sigma_{E} \in \mathcal{E}$. Cada rede particular é avaliada para $N_{\text {casos }}=20$ casos com localizações de RXs diferentes.

Avaliamos cada rede particular com a distância de erro médio total, em metros, $D_{\mathrm{e}}=\frac{1}{N_{\text {casos }}} \sum_{n=1}^{N_{\text {casos }}} d_{\mathrm{e}}^{(n)}$, em que $d_{\mathrm{e}}=\frac{1}{P} \sum_{k=1}^{P_{\mathrm{r}}} \gamma^{k}$ é a distância de erro médio para cada $\mathrm{RN}$, e $\gamma^{k}=\left\|\boldsymbol{v}_{\text {out }}^{k}-\hat{\boldsymbol{v}}_{\text {out }}^{k}\right\|$ é a norma da distância entre a posição do alvo teórica $\boldsymbol{v}_{\text {out }}^{k}$ e a estimada $\hat{\boldsymbol{v}}_{\text {out }}^{k}$ para o $k$-ésimo par. A Fig. 7 apresenta curvas de $D_{\mathrm{e}}$, para $\sigma_{E} \in \mathcal{E}$, vs neurônios $L \in \mathcal{L}$, com $P_{\mathrm{r}}=4000$, para sistemas de $M \in \mathcal{M} \mathrm{RXs}$, fixos e conhecidos (não apresentamos todas as curvas por limitação de espaço).

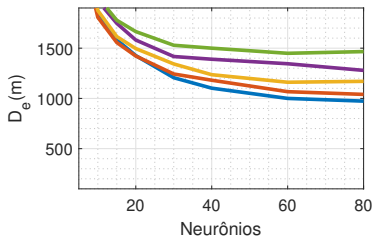

(a)

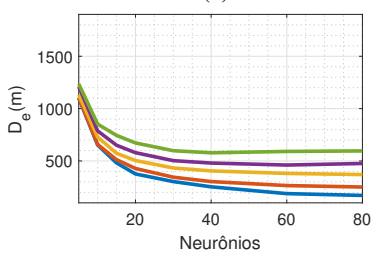

(c)

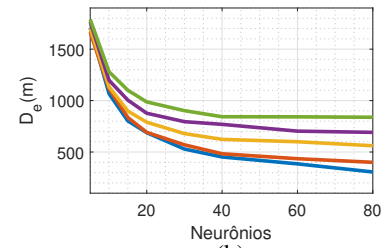

(b)

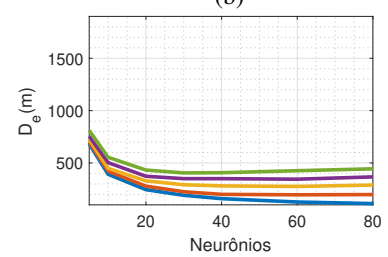

(d)

$$
-0 \%-1 \%-2 \%-3 \%-4 \%
$$

Fig. 8: $D_{\mathrm{e}}$ vs neurônios, $L$, para rede com RXs variáveis: $P_{\mathrm{r}}=8000$ : $P_{\text {trein }}=4000, P_{\text {teste }}=2000$ e $P_{\text {val }}=2000$ e $\sigma_{E}$ variando de $0 \mathrm{a}$ $4 \%$ de $\tau_{m}$. (a) $M=3$, (b) $M=4$, (c) $M=6$, (d) $M=10$.

Levando em conta a área onde o alvo pode estar localizado, consideramos uma rede otimizada quando $D_{\mathrm{e}} \leq 200 \mathrm{~m}$. Assim,

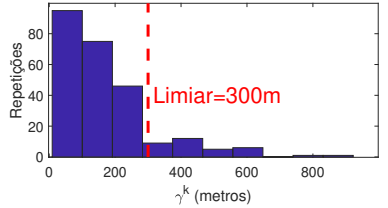

(a)

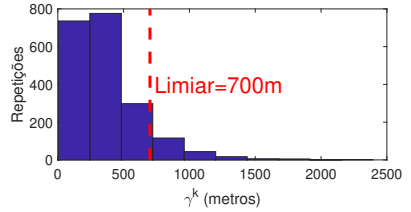

(b)
Fig. 9: Histograma da norma da distância entre a posição do objetivo teórica e a posição do objetivo estimada: $\gamma^{k}$ (metros): (a) Sistema com 6 RXs fixos da Fig. 6b. (b) Sistema com 6 RXs variáveis.

com $P_{\mathrm{r}}=400$ conseguimos sistemas ótimos até $\sigma_{E}=2 \% \tau_{m}$ $(M \geq 10, L \geq 12)$. Com $P_{\mathrm{r}}=1000$, obtêm-se sistemas até $\sigma_{E}=3 \% \tau_{m}(M \geq 15, L \geq 15)$. Com $P_{\mathrm{r}}=2000$, consegue-se sistemas até $\sigma_{E}=4 \% \tau_{m}(M \geq 15, L \geq 15)$ e com $P_{\mathrm{r}}=4000$ consegue-se sistemas até $\sigma_{E}=4 \% \tau_{m}(M \geq 10, L \geq 20)$.

Para RNs com RXs variáveis, fizemos experimentos para: $P_{\mathrm{r}} \in \overline{\mathcal{P}} \triangleq\{2000,4000,8000\}, M \in \overline{\mathcal{M}} \triangleq\{3,4,6,10\}$, $L \in \overline{\mathcal{L}} \triangleq\{5,6, \ldots, 80\}$ e $\sigma_{E} \in \mathcal{E}$. Não consideramos nestes experimentos possíveis erros nos posicionamentos dos RXs. Para cada caso particular, treinamos $N_{\text {casos }}=5$ redes com os mesmos pares para fazer uma avaliação média. Cada rede particular é avaliada com $D_{\mathrm{e}}$. A Fig. 8 apresenta curvas de $D_{\mathrm{e}}$, para $\sigma_{E} \in \mathcal{E}$, vs neurônios $L \in \overline{\mathcal{L}}$ para sistemas de $M \in \overline{\mathcal{M}}$ RXs, variáveis e conhecidos, com $P_{\mathrm{r}}=8000$.

Levando em conta a rede de RXs variáveis que tem uma complexidade maior, e a superfície de localização do alvo, consideramos uma rede razoável quando $D_{\mathrm{e}} \leq 400 \mathrm{~m}$. Desta maneira, verificamos que com $P_{\mathrm{r}}=2000$ não conseguimos sistemas razoáveis. Por outro lado, com $P_{\mathrm{r}}=4000$, conseguese sistemas até $\sigma_{E}=2 \% \tau_{m}(M \geq 10, L \geq 30)$. Finalmente, com $P_{\mathrm{r}}=8000$ conseguimos sistemas razoáveis até $\sigma_{E}=$ $3 \% \tau_{m}$ com $(M \geq 10, L \geq 60)$.

Na prática, os RXs representam o recurso mais caro da RN. Desta maneira, é melhor usar mais vetores na criação da rede. Para uma rede prática com RXs fixos, poderíamos considerar de 4 a 6 RXs com $P_{\mathrm{r}}=4000$ com $L=15$ a 20 neurônios. Já para uma rede com RXs variáveis, consideraríamos $6 \mathrm{RXs}$ com $P_{\mathrm{r}}=8000$ e $L=80$ neurônios.

\section{B. Pós-processamento da rede}

Após criada a RN, faz-se necessária uma crítica dos resultados. A crítica precisa ser feita individualmente, para cada rede. Por exemplo, no sistema com 6 RXs fixos da Fig. 6b, uma vez criada a rede, calculamos a norma da distância, $\gamma^{k}$, entre a posição do alvo teórica e a estimada do conjunto de validação. Neste caso $P_{\text {val }}=250$. No histograma de $\gamma^{k}$, apresentado na Fig. 9a, verificamos que a maior parte dos valores $(87,6 \%)$ estão concentrados entre 0 e $300 \mathrm{~m}$. $\mathrm{O}$ valor médio de $\gamma^{k}$ é $160 \mathrm{~m}$. Por outro lado, os valores mais afastados, pertencem a localizações onde foi complicado obter uma estimativa razoável. Estas posições complicadas, por observação gráfica, se apresentam quando $\gamma^{k} \geq 300 \mathrm{~m}$. Neste caso, das 250 posições avaliadas temos 31 posições complicadas.

Na Fig. 6b também ressaltamos as posições complicadas do alvo. Estas posições, de maneira geral não favorecidas pela geometria do sistema, podem facilmente ser afetadas pelo erro 


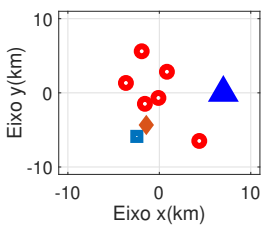

(a)

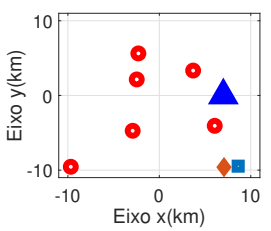

(c)

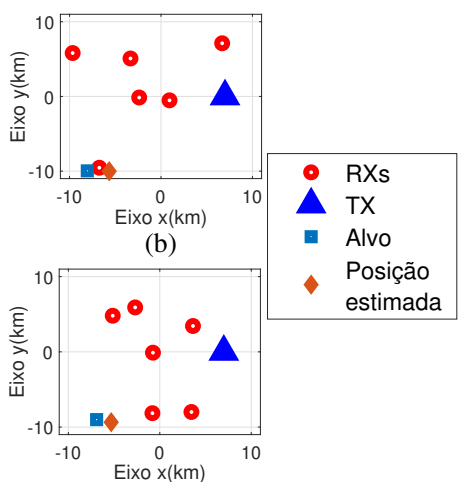

(d)
Fig. 10: Sistemas de localização com 6 RXs variáveis e uma posição de alvo onde $\gamma^{k} \geq 1200 \mathrm{~m}$ (localização complicada).

introduzido nas entrada. Tais posições, em sua maioria, como observadas na Fig. 6b, estão contornando a região interna entre os RXs e TX. Foram feitos experimentos para sistemas com diferentes localizações dos RXs e a conclusão foi similar.

Para o caso de sistema com RXs variáves, usamos como exemplo uma rede de $M=6 \mathrm{RXs}$ criada com $L=50$ neurônios, $P_{\mathrm{r}}=8000$ pares e $\tau_{m}=2 \% \tau_{m}$. Computamos $\gamma^{k}$ para o conjunto validação $\left(P_{\text {val }}=2000\right)$. O valor médio de $\gamma^{k}$ é $372 \mathrm{~m}$ e seu histograma é apresentado na Fig. 9b. A maior parte destes dados $(89,75 \%)$ estão concentrados entre 5 e 700 $\mathrm{m}$, determinando que valores de $\gamma^{k} \geq 700 \mathrm{~m}$ são considerados localizações complicadas (205 casos de um total de 2000). Na Fig. 10 apresentamos 4 casos mais críticos, $\gamma^{k} \geq 1200 \mathrm{~m}$, onde a geometria dos sistema com referência ao alvo não favorece uma boa estimativa da localização. Podemos observar que o alvo está afastado da região interna entre TX-RXs ou muito perto de um dos Rx. Uma análise mais profunda (fora do escopo deste trabalho) precisa ser feita para estes casos.

\section{Comparação com outros métodos}

As melhores redes com RXs fixos, criadas com $P_{\mathrm{r}}=4000$ pares, são usadas para comparar com os métodos: SI, SX [4] e NLCLS [7]. $N_{\text {casos }}=20$ casos de localizações de RXs diferentes são usados para obter o erro médio global $D_{\mathrm{e}}$ da rede neural e de tais métodos usando o grupo de vetores de validação. A TABELA III apresenta os resultados.

Claramente, as redes neurais com RXs fixos apresentam melhor precisão nas estimativas quando criadas com uma quantidade significativa de pares. O método NLCLS é o segundo método que apresenta melhores resultados tomando em conta que aplica uma restrição para diminuir a interferência do erro de $\tau_{m}$ na estimativa de localização. Por outro lado, fizemos comparações com RNs com RXs variáveis com melhores resultados $\left(N_{\text {rede }}=8000\right)$, considerando os mesmos valores de $M$ e $\sigma_{E}$ da TABELA II. Estas redes só têm melhor precisão e acurácia do que o método SX para os casos $\left(M=6, \sigma_{E}=3 \%\right),\left(M=10, \sigma_{E}=3 \%\right)$ e $\left(M=10, \sigma_{E}=3 \%\right)$, e do que o método SI para o caso $\left(M=10, \sigma_{E}=3 \%\right)$.

\section{CONCLUSÕES}

Para a aplicação sendo investigada, a RN feedforward com RXs fixos apresentou os melhores resultados, mesmo com
TABELA III: Erro médio global $D_{\mathrm{e}}$ em metros para a rede neural com RXs fixos e os métodos SI, SX e NLCLS

\begin{tabular}{|c|c|c|c|c|c|c|}
\hline \multicolumn{3}{|c|}{ Dados } & \multicolumn{4}{c|}{ Método } \\
\hline$M$-RXs & $\begin{array}{c}L \\
\text { neurônios }\end{array}$ & $\sigma_{E}$ & $\begin{array}{c}\text { Rede } \\
\text { Neural }\end{array}$ & SI & SX & NLCLS \\
\hline 4 & 15 & $1 \% \tau_{m}$ & $\mathbf{1 1 4 , 2 8}$ & 196,42 & 209,04 & 130,14 \\
\hline 4 & 15 & $2 \% \tau_{m}$ & $\mathbf{1 9 9 , 8 9}$ & 391,88 & 426,47 & 256,50 \\
\hline 6 & 20 & $2 \% \tau_{m}$ & $\mathbf{1 3 4 , 2 1}$ & 300,59 & 369,76 & 211,94 \\
\hline 6 & 20 & $3 \% \tau_{m}$ & $\mathbf{1 8 9 , 5 7}$ & 448,07 & 518,68 & 312,65 \\
\hline 10 & 20 & $3 \% \tau_{m}$ & $\mathbf{1 3 8 , 4 3}$ & 340,58 & 479,64 & 253,50 \\
\hline 10 & 20 & $4 \% \tau_{m}$ & $\mathbf{1 8 4 , 4 9}$ & 458,21 & 614,09 & 338,22 \\
\hline
\end{tabular}

presença de ruído. Os melhores sistemas, criados com 4000 pares (entrada-saída), apresentam os seguintes desempenhos de acordo com o número de RXs: para 4 RXs suporta erro gaussiano até $\sigma_{E}=2 \% \tau_{m}$, para 6 RXs até $\sigma_{E}=3 \% \tau_{m}$ e para $10 \mathrm{RXs}$ até $\sigma_{E}=4 \% \tau_{m}$ (com erro global $D_{\mathrm{e}} \leq 200 \mathrm{~m}$ ). Estes resultados foram melhores que os métodos SI, SX e NLCLS. Por outro lado, o sistema com RXs variáveis, tomando em conta a sua complexidade com o triplo de elementos de entrada, oferece os melhores resultados, com $P=8000$, para: 4 RXs até $\sigma_{E}=1 \% \tau_{m}, 6 \mathrm{RXs}$ até $\sigma_{E}=2 \% \tau_{m}$ e $10 \mathrm{RXs}$ até $\sigma_{E}=3 \% \tau_{m}$, (com erro global $\left.D_{\mathrm{e}} \leq 400 \mathrm{~m}\right)$.

No pós-treinamento, verificamos as posições do alvo que não estão favorecidas pela geometria do sistema e que são mais susceptíveis ao ruído. Trabalhos futuros poderiam explorar neurônios de base radial visando a atingir uma maior precisão nas estimativas destas posições. Também em pesquisas futuras, poderia ser investigada a robustez de uma RN quando temos ruído impulsivo, outliers, comum em aplicações práticas devido à possibilidade de multipercurso ou mesmo da falta de linha de visada direta para algum par TX-RX.

\section{REFERÊNCIAS}

[1] H. Griffiths, "Chapter 16 - Passive Bistatic Radar." Editors: N.D Sidiropoulos, F. Gini, R. Chellappa, S. Theodoridis, Academic Press Library in Signal Processing, Elsevier, vol. 2, 2014, pp. 813-855.

[2] R. S. Thomä, C. Andrich, G. D. Galdo, M. Dobereiner, M. A. Hein, M. Kaske, G. Schafer, S. Schieler, C. Schneider, A. Schwind, and P. Wendland, "Cooperative passive coherent location: A promising 5G service to support road safety," IEEE Communications Magazine, vol. 57, no. 9, pp. 86-92, Sep. 2019.

[3] M. Mellen and J. Raquet, "Closed-form solution for determining emitter location using time difference of arrival measurements," IEEE Transactions on Aerospace and Electronic Systems, vol. 39, no. 3, pp. 1056-1058, July 2003.

[4] M. Malanowski and K. Kulpa, "Two methods for target localization in multistatic passive radar," IEEE Transactions on Aerospace and Electronic Systems, vol. 48, no. 1, pp. 572-580, Jan. 2012.

[5] Z. Y. L. Jinga and L. Donghai, "Accurate single-observer passive coherent location estimation based on TDOA and DOA," Chinese Journal of Aeronautics, vol. 27, no. 4, pp. 913-923, Aug. 2014.

[6] S. Kaiser, A. Christianson, and R. Narayanan, "Multistatic Doppler estimation using global positioning system passive coherent location,' IEEE Transactions on Aerospace and Electronic Systems, vol. 55, no. 6 , pp. 2978-2991, Feb. 2019.

[7] D.P. Nicolalde-Rodríguez, J.A. Apolinário Jr., and W.A. Martins, "Robust passive coherent location via nonlinearly constrained least squares," 12th IEEE Latin American Symposium on Circuits \& Systems (LASCAS), Arequipa, Peru, Feb. 2021, pp. 1-4.

[8] W. Guan, B. Lu, B. Li and P. Jiang, "A TDOA localization algorithm based on Elman neural network for cellular networks," Information Technology Journal, vol. 12, no. 23, pp. 7143-7147, 2013.

[9] S. Pak, B.K. Chalise and B. Himed, "Target localization in multi-static passive radar systems with artificial neural networks," International Radar Conference (RADAR), Toulon, France, Sep. 2019, pp. 1-5.

[10] S. Haykin, Neural Networks and Learning Machines. Pearson - Prentice Hall, 3rd edition, 2008. 